\title{
EDITORIAL
}

\section{LAS DESIGUALDADES DE GÉNERO EN SALUD: RETOS PARA EL FUTURO}

\section{Carme Borrell $(1,2,3)$ y Lucía Artazcoz $(1,2)$}

(1) Agència de Salut Pública de Barcelona.

(2) CIBER Epidemiología y Salud Pública (CIBERESP).

(3) Universitat Pompeu Fabra.

A pesar de la progresiva utilización del término "género" en la literatura de las ciencias de la salud, aún existe una confusión generalizada y los conceptos "sexo" y "género" se usan a menudo indistintamente.

El género se refiere a un constructo social basado en las convenciones culturales, actitudes y relaciones entre hombres y mujeres $^{1-5} \mathrm{y}$, por lo tanto, no es una categoría estática sino que se produce y reproduce a través de las acciones de las personas pudiendo cambiar de una sociedad a otra y también a lo largo de la historia. En cambio el sexo se refiere a las diferencias físicas, anatómicas y fisiológicas de hombres y mujeres. Tanto el género como el sexo se relacionan con la salud y lo hacen de forma simultánea, ya que las personas no viven siendo de un "género" o de un sexo", sino de ambos a la vez².

Las diferencias de sexo y de género determinan diferencias en los determinantes de la salud, la vulnerabilidad, la naturaleza, severidad y frecuencia de los problemas de salud, la forma en la que se perciben

Correspondencia:

Carme Borrell

Agència de Salut Pública de Barcelona

Pl. Lesseps 1

08023 Barcelona

cborrell@aspb.cat los síntomas, la utilización y la accesibilidad de los servicios sanitarios, el esfuerzo diagnóstico y terapéutico, el cumplimiento del tratamiento y de los mensajes preventivos y el pronóstico de los problemas de salud entre hombres y mujeres ${ }^{6}$.

\section{¿QUÉ SIGNIFICA INCORPORAR \\ LA PERSPECTIVA DE GÉNERO EN EL ANÁLISIS DE LAS DESIGUALDADES EN LA SALUD?}

Introducir la perspectiva de género en el abordaje de los problemas de salud requiere la consideración de las diferencias en la socialización de mujeres y hombres, las cuales determinan distintos valores, actitudes y conductas, las desigualdades en el poder y en el acceso a los recursos y la profunda división sexual del trabajo. Sin embargo, con frecuencia la introducción de la sensibilidad de género en los estudios de los determinantes sociales de la salud se ha basado en muestras constituidas exclusivamente por mujeres, pero el sexo y el género son también determinantes de la salud de los hombres ${ }^{7-9}$. Así, la sistemática subordinación de las mujeres (patriarcado) requiere, entre otras cosas, la demostración de la fortaleza física y la exposición a riesgos para la salud por parte de los hombres. Ellos deben mostrarse fuertes y saludables 
y no evidenciar debilidades, lo cual está implícito en la construcción del género masculino (masculinidad). El desarrollo de una identidad masculina heterosexual tradicional significa, por ejemplo, la adopción de hábitos no saludables ${ }^{10}$. Podemos citar los siguientes ejemplos: el mayor consumo de tabaco y alcohol por parte de los hombres, la adopción de conductas de riesgo en la conducción de vehículos, la menor realización de prácticas preventivas, como por ejemplo la aplicación de crema protectora solar, el menor reconocimiento de sus problemas de salud y, por lo tanto, el retraso en las visitas a los profesionales sanitarios, etc. Estos comportamientos de riesgo asociados a la masculinidad tradicional son responsables en buena medida de la menor esperanza de vida de los hombres. En nuestro medio, un reto para el futuro es analizar la repercusión en la salud de la masculinidad.

Por tanto, la descripción y la investigación sobre las diferencias según sexo y de las desigualdades según género en la salud deben tener en cuenta tanto a las mujeres como a los hombres y, siempre que sea posible, deben realizarse análisis separados por sexo para poder entender la naturaleza del género como un concepto multidimensional. El hecho de analizar conjuntamente a hombres y mujeres, es decir, considerar la variable sexo como confundidora, sin tener en cuenta la posible existencia de una interacción, significa asumir que los determinantes de la salud son los mismos para los dos sexos pese a las notables diferencias biológicas y de género que explican diferencias en un amplio abanico de dimensiones de la salud entre hombres y mujeres ${ }^{11}$. Sin embargo, no es infrecuente encontrar estudios e informes que no separan los resultados para hombres y mujeres, o al menos no lo hacen sistemáticamente, incluso en temas con diferencias de género tan marcadas como el consumo de drogas ${ }^{12}$. Esta práctica debe erradicarse no sólo en aras del rigor científico, sino teniendo en cuenta, además, que la Ley de igualdad de mujeres y hombres ${ }^{13}$ aprobada en marzo de 2007 señala que "el tratamiento de los datos contenidos en registros, encuestas, estadísticas... deben permitir el análisis de género, incluyendo, siempre que sea posible, su desagregación por sexo".

\section{LA DISCRIMINACIÓN, EL PODER Y SU IMPACTO EN LA SALUD}

Relacionado con los conceptos de sexo y género, está el sexismo o discriminación por sexo, que se refiere a las relaciones de género injustas basadas en prácticas institucionales e interpersonales donde miembros de un grupo dominante (habitualmente los hombres) adquieren privilegios a base de subordinar a otros géneros (habitualmente las mujeres) y justifican estas prácticas mediante ideologías de superioridad o diferencias $^{2}$. La discriminación puede ser legal, como por ejemplo lo fueron las leyes que impedían votar a las mujeres, o ilegal. Se perpetúa a través de muchos actores, tanto del estado y sus instituciones, como de organizaciones privadas o a través de los individuos ${ }^{14}$. La discriminación afecta la salud en distintos grados, siendo el máximo nivel de expresión la violencia física y mental, como la violencia de género responsable de miles de muertes en todo el mundo ${ }^{15,16}$. Existen aún pocos estudios sobre la repercusión en la salud de la discriminación ${ }^{17}$, sobre todo, en nuestro país ${ }^{18}$, hecho que podrá ser parcialmente solventado debido a la inclusión de preguntas sobre discriminación percibida en la Encuesta Nacional de Salud de $2006^{19}$.

En la mayoría de las sociedades industrializadas se produce una desigual distribución en función del sexo en el mundo de la producción (económico-mercantil) y el mundo de la reproducción (ámbito doméstico-familiar que garantiza el sostenimiento de la vida humana) que es discriminatoria para las mujeres, lo que se traduce en una desigual repartición del poder entre 
hombres y mujeres ${ }^{20}$. Estas discriminaciones deberán tenerse en cuenta al estudiar las desigualdades en salud según género y las diferencias según sexo. Así, los hombres realizan mayoritariamente el trabajo productivo fuera de casa que es considerado fundamental y que es reconocido socialmente, y las mujeres se encargan básicamente de las tareas relacionadas con el cuidado de las personas, que son consideradas complementarias y sin tanto valor social. La progresiva incorporación de las mujeres al mercado laboral ha estado marcada por la existencia de una fuerte segregación laboral según género, tanto horizontal (las mujeres se concentran en unos pocos sectores de actividad económica, sobre todo en los servicios) como vertical, ya que las mujeres ocupan la mayoría de trabajos menos cualificados y con menor autoridad. Debido a la segregación de género del mercado laboral, un sector importante de los hombres se concentran en ocupaciones con alto riesgo de accidentes laborales ${ }^{3}$. Por otro lado, el desempleo, los contratos precarios y los salarios bajos son más frecuentes en las mujeres.

El artículo de Carrasco-Portiño et al. ${ }^{21}$ que se presenta en este número de la Revista Española de Salud Pública, describe el Índice de Desarrollo de Género en el Estado español y sus Comunidades Autónomas, índice construido a partir de las desigualdades entre hombres y mujeres en la esperanza de vida, el nivel de ingresos y en la educación. Mientras que la esperanza de vida es más favorable para las mujeres, los otros dos indicadores son más favorables para los hombres, aunque la situación ha cambiado entre 1990 y 2000 , sobre todo en lo que respecta a la educación, ya que en el año 2000 las diferencias entre hombres y mujeres son menores.

Otro ejemplo de la discriminación de las mujeres es su sistemática exclusión de los ensayos clínicos o de estudios relevantes sobre la salud y la enfermedad, asumiendo que los resultados obtenidos al estudiar a los hombres pueden ser extrapolados a las mujeres, cuando no siempre es asíi ${ }^{22}$. El artículo de Laguna y de Andrés ${ }^{23}$, también en este número de la Revista Española de Salud Pública, pone en evidencia que la mayoría de los informes finales de ensayos clínicos recibidos en la Agencia Española de Medicamentos y Productos Sanitarios a comienzos de 2007 (justo cuando se aprobó la Ley de Igualdad) no desagregaban la información por sexo. Este estudio puede servir como base en el futuro para evaluar la Ley de Igualdad respecto a este tema.

\section{LAS DESIGUALDADES SEGÚN GÉNERO Y CLASE SOCIAL}

Con frecuencia, los estudios sobre desigualdades en salud según género se han realizado de forma paralela a los estudios de desigualdades según clase social, no existiendo una visión integradora de ambas líneas de trabajo. No es posible comprender las desigualdades en salud según género sin tener en cuenta las desigualdades según clase social, ya que las personas son de un género y de una clase social. Así por ejemplo, a pesar de que la salud percibida de las mujeres del Estado español es peor que la de los hombres, la salud percibida de una mujer de clase privilegiada es mejor que la de un hombre trabajador manual ${ }^{24}$.

Recientemente, algunos autores y autoras han empezado a tener en cuenta simultáneamente los ejes de desigualdad de género y clase social ${ }^{25,26}$, a pesar de que no existe un consenso claro de cuál es la medida de clase social a utilizar para las mujeres $^{27,28}$. Así, se ha comenzado a analizar el impacto en la salud de las distintas posiciones en la sociedad y de los múltiples roles realizados por las mujeres y cómo este impacto varía en función de la clase social y de la privación material de la mujer ${ }^{29}$. En nuestro medio, en la última década, han empezado a publicarse algunos estudios 
que intentan integrar los dos ejes de desigualdad. Son fundamentalmente investigaciones que analizan la salud de mujeres y hombres según su clase social teniendo en cuenta distintos grupos de análisis en función de la situación laboral, los trabajos realizados, el estado civil o las demandas familiares ${ }^{30,31}$. En el futuro no sólo será importante tener en cuenta los ejes de desigualdad de clase social y género, sino también el país de origen de las personas debido a la importante inmigración que ha existido en nuestro país en los últimos años $^{32}$.

\section{LAS POLÍTICAS DE SALUD Y LAS DESIGUALDADES DE GÉNERO}

Por último, es necesario señalar que las políticas de salud también deben tener en cuenta las desigualdades de género, lo que se debe visualizar en los siguientes aspectos $^{33}$ : en la construcción social de los problemas de salud, en la toma de decisiones y en la formulación, implantación y evaluación de las políticas de salud. Tanto la Ley de Violencia de Género de $2004^{34}$, como la Ley de Igualdad entre mujeres y hombres de $2007^{13}$ representan un avance en este aspecto. La primera ya ha empezado a ser evaluada ${ }^{35}$ y la segunda deberá serlo en el futuro. Además, será necesario que los Planes de salud de las Comunidades Autónomas avancen en el enfoque de género ya que, en general, existe un mayor contenido en la descripción de la situación de la salud y sus determinantes que en la formulación de objetivos e indicadores de evaluación ${ }^{36}$.

\section{BIBLIOGRAFÍA}

1. Krieger N. A glossary for social epidemiology. J Epidemiol Community Health. 2001; 55: 693700.

2. Krieger N. Genders, sexes, and health: what are the connections--and why does it matter? Int J Epidemiol. 2003; 32: 652-7.
3. Courtenay WH. Constructions of masculinity and their influence on men's well-being: a theory of gender and health. Soc Sci Med. 2000; 50: 1385401.

4. Doyal L. Sex and gender: the challenges for epidemiologists. Int J Health Serv. 2003; 33: 569-79.

5. Barres BA. Does gender matter? Nature. 2006; 442: 133-6.

6. Borrell C, Artazcoz L (coord.). 5 a Monografía de la SEE: Investigación sobre género y salud. Barcelona: Sociedad Española de Epidemiología; 2007.

7. Courtenay WH. Constructions of masculinity and their influence on men's well-being: a theory of gender and health. Soc Sci Med. 2000; 50: 1385-401.

8. Moynihan C. Theories in health care and research: theories of masculinity. BMJ. 1998; 317: 1072-5.

9. Doyal L. Sex, gender, and health: the need for a new approach. BMJ. 2001; 323: 1061-3.

10. Courtenay WH, Keelin RP. Men, Gender, and Health: Toward an Interdisciplinary Approach. J Am Coll Health. 2000; 48: 243-246.

11. Kunkel SR, Atchley RC. Why gender matters: being female is not the same as not being male. Am J Prev Med. 1996; 12: 294-6.

12. Observatorio Europeo de las Drogas y las Toxicomanías. Informe anual 2007. El problema de la drogodependencia en Europal. Luxemburgo: Oficina de Publicaciones Oficiales de las Comunidades Europeas. 2007.

13. Ley orgánica $3 / 2007$, de 22 de marzo, para la igualdad efectiva de mujeres y hombres. BOE núm 71, 23/03/2007.

14. Krieger N. Discrimination and health. En: Berckman L, Kawachi I. Society and health. Oxford: Oxford University Press: 2000.p. 36-75.

15. WHO Multi-country Study on Women's Health and Domestic Violence against Women. WHO; 2005. Disponible en: http://www.who.int/gender/violence/who_multicountry_study/en/index.ht $\mathrm{ml}$.

16. Blanco P, Ruiz-Jarabo C, Garcia de Vinuesa L, Martin-Garcia M. La violencia de pareja y la salud de las mujeres. En: Borrell C, García-Calvente MM, Martí-Boscà V (eds). Informe SESPAS 2004. La salud pública desde la perspectiva de género y clase social. Gac Sanit. 2004; 18 (Suppl 1):182-8 
17. Krieger N, Smith K, Naishadham D, Hartman C, Barbeau EM. Experiences of discrimination: validity and reliability of a self-report measure for population health research on racism and health. Soc Sci Med. 2005; 61: 1576-96.

18. Pantzer K, Rajmil L, Tebe C, Codina F, Serra-Sutton V, Ferrer M, Ravens-Sieberer U, Simeoni MC, Alonso J. Health related quality of life in immigrants and native school aged adolescents in Spain. J Epidemiol Community Health. 2006; 60: 694-8.

19. Rohlfs I, Borrell C, Artazcoz L, Escribà-Agüir V. The incorporation of gender perspective into Spanish health surveys. J Epidemiol Community Health. 2007; 61 (Suppl 2): ii20-25.

20. Artazcoz L, Borrell C, Cortès I, Escribà-Agüir V, Cascant L. Occupational epidemiology and work related inequalities in health: a gender perspective for two complementary approaches to work and health research. J Epidemiol Community Health. 2007; 61 (Suppl 2): ii39-45.

21. Carrasco-Portiño M, Ruiz-Cantero MT, Gil-González D, Álvarez-Dardet Díaz C, TorrubianoDomínguez J. Epidemiología de las desigualdades del desarrollo de género en España (1990-2000). Rev Esp Salud Pública. Rev Esp Salud Pública. 2008; 82:283-99.

22. Ruiz MT, Verbrugge LM. A two way view of gender bias in medicine. J Epidemiol Community Health. 1997; 51:106-9.

23. Laguna Goya N, de Andrés Rodríguez-Trelles F. Participación de las mujeres en los ensayos clínicos. Estudio inicial en archivos de la agencia española de medicamentos y productos sanitarios Rev Esp Salud Pública. 2008; 82:343-50.

24. Rodríguez-Sanz M, Carrillo Santisteve P, Borrell C. Desigualdades sociales en la salud, los estilos de vida y la utilización de servicios sanitarios en las Comunidades Autónomas, 1993-2003. Madrid: Observatorios de Salud de la Mujer y del Sistema Nacional de Salud. Dirección General de la Agencia de Calidad del Sistema Nacional de Salud. Ministerio de Sanidad y Consumo, 2005. Disponible en: http://www.msc.es/ organizacion/sns/planCalidad-SNS/pdf/equidad/Desigualdades_sociales_salud_y_SS.pdf (consultado el 1-6-2008)

25. Bartley M. Measuring women's social position: the importance of theory. J Epidemiol Community Health. 1999; 53: 601-2.
26. Emslie C, Hunt K, Macintyre S. Problematizing gender, work and health: the relationship between gender, occupational grade, working conditions and minor morbidity in full-time bank employees. Soc Sci Med. 1999;48:33-48.

27. Borrell C, Rohlfs I, Artazcoz L, Muntaner C. Desigualdades en salud según la clase social en las mujeres. ¿Cómo influye el tipo de medida de la clase social? Gac Sanit. 2004; 18 ( Suppl 2):75-82.

28. Krieger N, Chen JT, Selby JV. Comparing individual-based and household-based measures of social class to assess class inequalities in women's health: a methodological study of 684 US women. Epidemiol Community Health. 1999; 53: 612-23.

29. Arber S, Khlat M. Introduction to "social and economic patterning of women's health in a changing world". Soc Sci Med. 2002; 54: 643-7.

30. Artazcoz L, Borrell C, Benach J. Gender inequalities in health among workers: the role of family demands. J Epidemiol Community Health. 2001; 55: 639-47.

31. Borrell C, Muntaner C, Benach J, Artazcoz L. Social class and self-perceived health status among men and women: what is the role of work organization, household material standards and household labour. Soc Sci Med. 2004; 58: 1869-87.

32. La salut de la població immigrant a Barcelona. Barcelona: Agència de Salut Pública de Barcelona; 2008.

33. Peiró R, Vives C, Alvarez-Dardet C, Más R. El análisis de políticas con enfoque de género y salud. En: Borrell C, Artazcoz L (coord.). $5^{\text {a }}$ Monografía de la SEE: Investigación sobre género y salud. Barcelona: Sociedad Española de Epidemiología; 2007.

34. Ley orgánica $1 / 2004$, de 28 de diciembre, de Medidas de Protección Integral contra la Violencia de Género. BOE núm 313, 29/12/2004.

35. Vives-Cases C, Torrubiano-Domínguez J, Álvarez-Dardet C. Distribución temporal de las denuncias y muertes por violencia de género en España en el período 1998-2006. Rev Esp Salud Pública. 2008; 82: 91-100.

36. Peiró R, Ramón N, Álvarez-Dardet C, Colomer C, Moya C, Borrell C, y col. Sensibilidad de género en la formulación de planes de salud en España: lo que pudo ser y no fue. Gac Sanit. 2004; 18 (Supl 2): $36-46$. 
